\title{
Digital biofabrication to realize the potentials of plant roots for product design
}

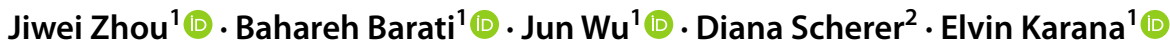

Received: 11 June 2020 / Accepted: 20 July 2020 / Published online: 4 September 2020

(c) The Author(s) 2020

\begin{abstract}
Technological and economic opportunities, alongside the apparent ecological benefits, point to biodesign as a new industrial paradigm for the fabrication of products in the twenty-first century. The presented work studies plant roots as a biodesign material in the fabrication of self-supported 3D structures, where the biologically and digitally designed materials provide each other with structural stability. Taking a material-driven design approach, we present our systematic tinkering activities with plant roots to better understand and anticipate their responsive behaviour. These helped us to identify the key design parameters and advance the unique potential of plant roots to bind discrete porous structures. We illustrate this binding potential of plant roots with a hybrid 3D object, for which plant roots connect 600 computationally designed, optimized, and fabricated bioplastic beads into a low stool.
\end{abstract}

Keywords Plant roots $\cdot$ Biodesign $\cdot$ Digital biofabrication $\cdot$ Material-driven design $\cdot$ Living organisms

\section{Introduction}

The cross-fertilization of biology with design and engineering offers new sustainable solutions and diverse forms of expressions for product design and fabrication [1-4]. This emerging practice suggests that the product is a co-creation of humans and living organisms, such as algae, fungi, bacteria and plants, in which the organisms might contribute as building blocks, material sources, energy generators and more [1]. Technological and economic opportunities, alongside its ecological benefits, point to biodesign as a new

Jiwei Zhou

jiwei.zhou@tudelft.nl

Bahareh Barati

b.barati@tudelft.nl

Jun Wu

j.wu-1@tudelft.nl

Diana Scherer

Interwoven.scherer@gmail.com

http://dianascherer.nl/

Elvin Karana

e.karana@tudelft.nl

1 Faculty of Industrial Design Engineering, Delft University of Technology, Delft, The Netherlands

2 Amsterdam, The Netherlands industrial paradigm for the fabrication of products in the twenty-first century [5-8].

Biodesign, within the context of product design, builds upon the relatively established field of biofabrication, which has a long history in biomedical science and engineering [5, 9-12].

Researchers have achieved to embed cells of microorganism, animal and plant origins into a variety of scaffold using digital fabrication technologies (e.g. [4, 13-18]). Today, potential applications of biodesign vary from biological energy sources (e.g. microbial fuel cells) to bio (-degradable) materials, such as fungi-based leather (e.g. https://www. mycoworks.com) and oil-free plastic and foam alternatives (e.g. https://www.bloomtreadwell.com).

While the majority of the biodesign projects are still at an experimental scale, recent initiatives of biodesign companies such as Ecovative, MycoWorks, MOGU and Modern Meadow for scaling-up are promising. In the fabrication of sustainable material alternatives for product design, many of the current initiatives focus on bacteria and fungi. An exception to this is a UK-based company, Fullgrown, which cultivates trees into wooden furniture by a self-developed process relying on traditional horticultural techniques such as grafting, pruning and espaliering. Fullgrown's commission-based business model allows them to deliver the grown products by trees in $6-10$ years. 
For thousands of years, plants have been (cultivated and) used as material sources by humans, resulting in a well-established repertoire of techniques and tools. Nevertheless, when it comes to biodesign-as also evident from the Fullgrown example-the transformation of plant or plant parts into products can be very lengthy. Therefore, despite the familiarity of the organism for humans, plants and plant parts have been less explored in biodesign, compared to other organisms. Addressing this gap, in this paper, we explore the potentials of plant roots for product design. In particular, we demonstrate the possibility of creating self-standing 3D structures by integrating computational design tools in designing with plant roots.

The potentials of living plants as (inter)active beings in the design of interactive products have long been explored by human-computer interaction (HCI) communities as [19-27]. In particular, the ability of plants to respond to the changes in the environment has inspired scholars to incorporate them in sensing devices [28-30], information outputs [31-35] and self-nurturing systems [36-39].

Embarking on a more artistic endeavour and fascinated by the intelligence and behaviour of plant roots, Diana Scherer has explored the material ability of plant roots at the seedling stage to create an unprecedented textile-like material, Interwoven. Through a self-developed technique, aided by digital fabricated templates which guides the growth of plant roots (patent pending), the artist directs plant roots into geometric patterns found in nature, like honeycomb structures, or foliate designs reminiscent of Middle Eastern arabesques, shown in Fig. 1. The process takes 1-2 weeks to complete.

Scherer's work illustrates that roots are not only productive means but also intelligent agents that respond to and adapt actively and dynamically to their environment. Yet, in order to further advance their applications in product design, there is a need to systematically understand plant root behaviour and explore new fabrication parameters. For instance, the digitally fabricated piece, which is used primarily to create templates for controlling the organism's growth, can be a part of the final artefact, a direction that has not yet been explored.
In collaboration with Diana Scherer, our work contributes to the understanding of plant root in designing and fabricating 3D objects with the aid of computational tools. The potential lies in the following aspects: (1) speed: In 1 or 2 weeks, oat roots are able to grow to around $120 \mathrm{~mm}$ according to our experiment. Therefore, we argue that plant roots have the potential to fabricate a number of low-height products at a high efficiency (compared to a tree stem). (2) $3 D$-form ability: we show the potential of plant roots for fabricating 3D forms. (3) glue-ability: Our work demonstrates the potential of roots to connect discrete computationally designed, optimized and fabricated beads into a $600 \mathrm{~mm}$ by $600 \mathrm{~mm}$ 3D mass. In this project, rather than creating boundaries between the living organisms and manmade materials, we progressively explored a symbiotic approach where the biologically and digitally designed materials provide each other with structural stability.

\section{Background}

\section{Digital biofabrication}

In engineering, computational design methods such as topology optimization have been developed to create lightweight and biomimetic structures [40-45] in a manner analogous to bone remodelling. Furthermore, parametric design allows to create materialized patterns that vary in structural properties, i.e. functionally graded meta-materials. In biodesign, coupling biological principles with advanced computational technologies, i.e. digital biofabrication [4], can help stretch the possibilities of what living organisms can offer for design. For example, digital fabrication has proved to be useful in producing detailed structures and thus creating a fine-tuned habitat for living organisms in a number of projects, e.g. 3D-printed irrigated green wall (https://arche 1lo.com/news/the-worlds-first-3d-printed-irrigated-green -wall) where agent-based modelling [46] is used to design the geometry of the habitat of plants. Another example to this is the Mycelium Chair, by designer Eric Klarenbeek, who 3D-printed straw (where mycelium grows) and a PLA
Fig. 1 Interwoven, plant roots are grown into intricate, textilelike materials (Diana Scherer: http://dianascherer.nl. Accessed 9 June 2020)

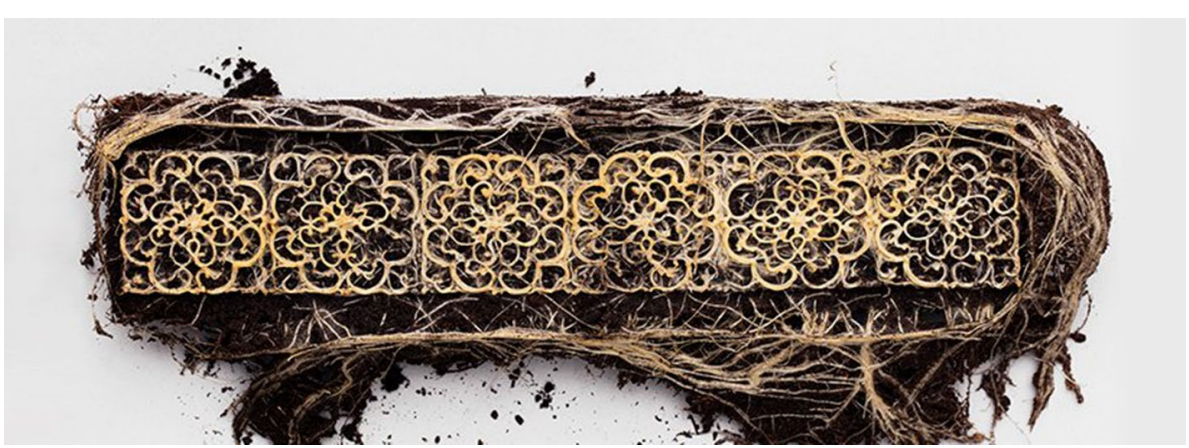


shell as a house for mycelium growth (https://www.dezee n.com/2013/10/20/mycelium-chair-by-eric-klarenbeek-is3d-printed-with-living-fungus/).

Other researchers have looked into the possibilities of computational design tools, in particular digital fabrication, to achieve nature-inspired and hybrid (of artificial and living) scaffolds. In the example of "Silk Pavilion" [47], the skeleton has been created with $\mathrm{CNC}$ and robotic arm, and silkworms were then placed onto the skeleton and produce silk on the existing structure (Fig. 3 Silk Pavilion made with silkworms (left), by MIT Media Lab; silkworm fabricating silk on the CNC scaffold (right) (https://www.dezee n.com/2013/06/03/silkworms-and-robot-work-together-toweave-silk-pavilion/). The behaviour of silkworms has been studied systematically for the fabrication of Silk Pavilion [48]. Likewise, the BioLogic project by the MIT Tangible Media Group [49] explores responsive clothing, enabled by dozens of tiny triangular flaps that react to heat and humidity due to the single-cell organisms 3D-printed on the fabric.

In the case of Interwoven by Diana Scherer, the digital tools are used to design and fabricate the 2D templates which direct the root plants to form a predefined patterns and a textile-like material. Thus, the digitally fabricated parts are only used to give the plant root material its final shape, while they could support the structural stability of the material if they were integrated into the material composition, as shown in Mycelium Chair and Silk Pavilion. In both examples, the resulting artefacts are hybrid/composite assemblies of living organisms and digital technologies. However, both of the cases have self-standing scaffolds that contain the organisms. On the contrary, our design utilizes the glue-ability of plant roots to bind discrete computationally optimized and fabricated beads and compound a stable structure.

In this paper, we focus on the design and optimization of the templates to create 3D structures, either by giving emphasis to the "process" design (e.g. turning the mould during the growth), or reinterpreting the template as a 3D-printed structure which ultimately builds a composite material together with plant roots. Hence, $3 D$ form-ability and structural stability lead our tinkering activities throughout the design process. Our work is a first step towards understanding the behaviour of roots for design.

\section{Root behaviour in response to environmental stimuli}

The successful development of a plant depends on the correct positioning of the seedling organs towards gravity, ensuring that the plant anchors its roots in the soil (providing nutrients and water) and adjusts light-harvesting shoot organs optimally above ground [50]. Roots do not passively grow, but move and observe [51]. Their movements allow them to better search for food and space to live and adapt intelligently to their environment [52]. In order to anchor the plant deeper underground through root movement, a type of tissue covering the tip of roots, called the root cap or calyptra, perceives gravity and direct root growth and development with regulatory processes of hormone network [53]. "Artificial gravity" [51], for instance caused by centrifugal force, determines the root direction in the same way as natural gravity does [54].

Moreover, roots twist their forms in response to the form and direction of the barriers faced in the growing direction. For instance, a tilted barrier leads to an in-plane coiling of the root, like waving, through actively twisting and passively directed by gravity [55].

Other important variables influencing the root behaviour and the properties of the final outcome (e.g. Interwoven) are type and amount of growing media and water [56]. As observed by Scherer and emphasized in the literature, a certain level of drought helps roots elongation $[56,57]$, while too much water slows down their process, "making the roots lazy" [Scherer, personal communication]. In order to create intricate and delicate patterns from roots, Scherer would leave the growing media (coco coir or soil) relatively dry to have longer roots (Fig. 2).

As presented above, a lot is known about how the plant root behaviour changes in response to environmental stimuli. Nevertheless, it remains unclear how and to what extent the root growth can be intentionally steered towards expressive and functional 3D forms/products. It is envisioned that digital design tools that support the creation of dedicated habitat for plants would facilitate this investigation.

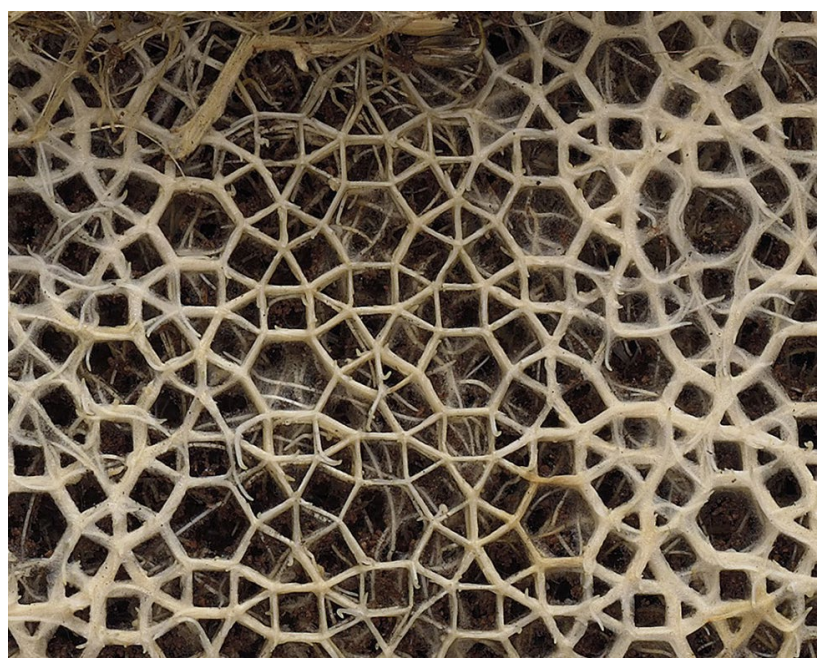

Fig. 2 Intricate and delicate patterns grown by plant roots in coco coir in a relatively drier condition to achieve longer roots (Diana Scherer: http://dianascherer.nl. Accessed 9 June 2020) 
Fig. 3 Taxonomy to support tinkering activities with plant roots

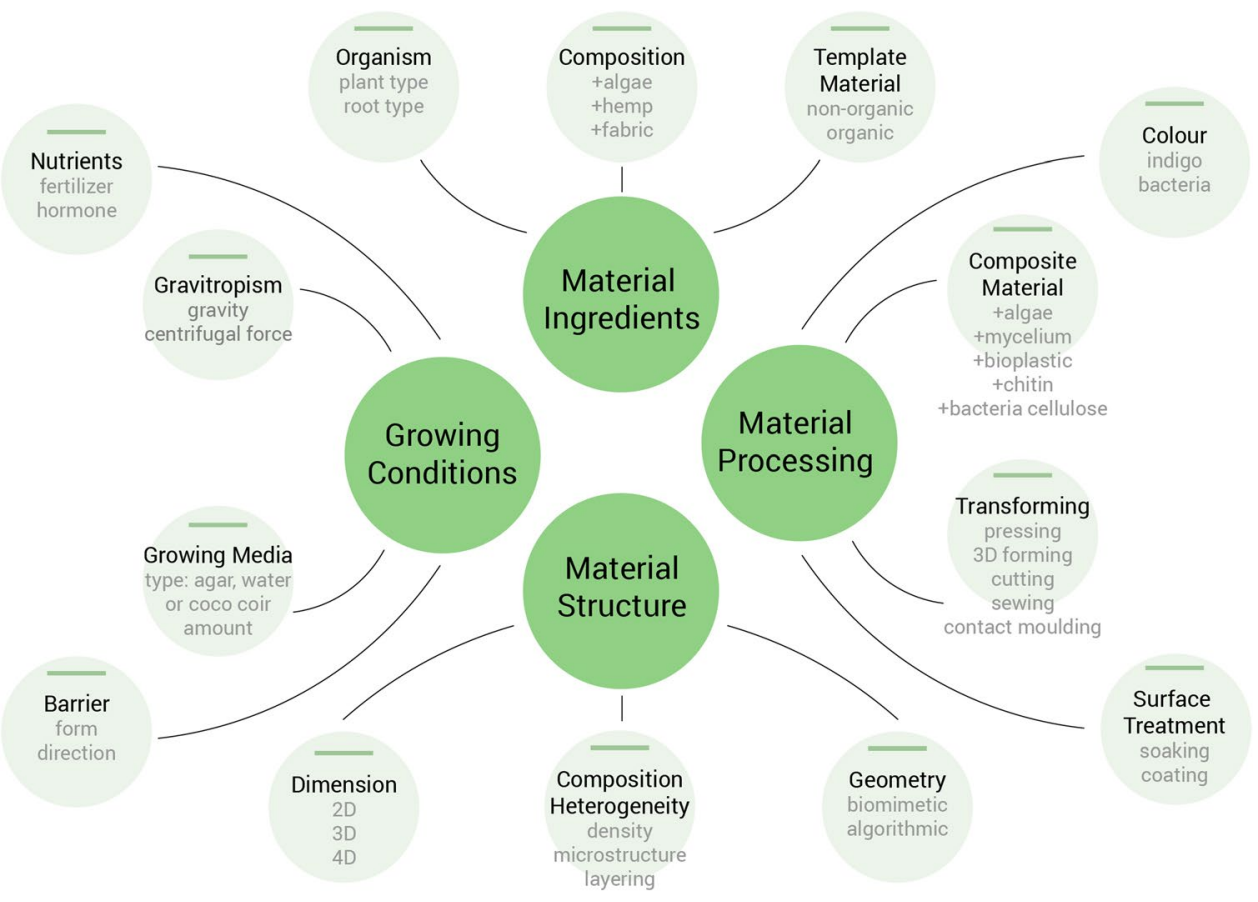

\section{Designing with plant roots: a case study}

In this section, we will present a case study focusing on plant roots. The design project was conducted at Material Experience Lab in Faculty Industrial Design Engineering in Delft University of Technology, in collaboration with the artist, Diana Scherer to address the identified knowledge gap in the literature concerning the creation of 3D structures. Accordingly, the aim of the project was set to "explore the potentials of plant roots for product design, with a particular emphasis to the structure stability and 3D-form ability of root materials".

\section{Research methodology}

The paper explains our material-driven design approach [58], combining systematic tinkering and sample creation, and elaborates on our findings in terms of (novel) affordances/potentials [59] of plant roots for digital biofabrication. Material-driven design (MDD) is a design approach to the exploration and capitalization of materials potential [58], which extends beyond the possibilities of materials for intended form, function and experience, to process-abilities that are perceived when tinkering with the material [59]. In working with plant roots, tinkering helped us to reduce the degree of uncertainty, which mainly derives from unpredictability and context dependency of living organisms $[4,60]$. By mapping out our findings of the root behaviour under various growing conditions to biological and computational design parameters in the growing design space, our work provides practical insights on creating 3D products with plant roots.

Before starting the tinkering activities, following the material-driven design method [58] we first created a taxonomy [60] which maps the key elements and relationships in growing plant roots (see Fig. 3).

The taxonomy consists of four main sections that include but not limited to the listed adjustable parameters: (1) Material ingredients, which are elements in the composition of the resulting material, including the organism used for the fabrication (different types of plants and roots), composition concerning what other materials (e.g. algae, hemp, etc.) are used in the growing process, and the template material (organic and non-organic) is used for guiding the growth. (2) Growing conditions encompass growth boosting nutrients (e.g. fertilizer and hormone), gravitropism (influencing growing direction), the type and amount of growing media and the form and direction of barriers roots face in their growing direction. (see "Root intelligence" section) (3) Material structure can have variables in dimension (2D to 4D), composition heterogeneity (density, local microstructure and layering structure) and geometry (biomimetic, algorithmic). (4) Material processing refers to the post-processing of the harvested biomass, including dyeing, compositing with other bio-based materials, transforming and surface treatment like coating. The above sections and parameters are closely interlinked with each other. For instance, the manipulation of barriers might lead to change in composition heterogeneity. Through manipulating one or more parameters, the resulting material sample may have different technical properties and experiential qualities. 


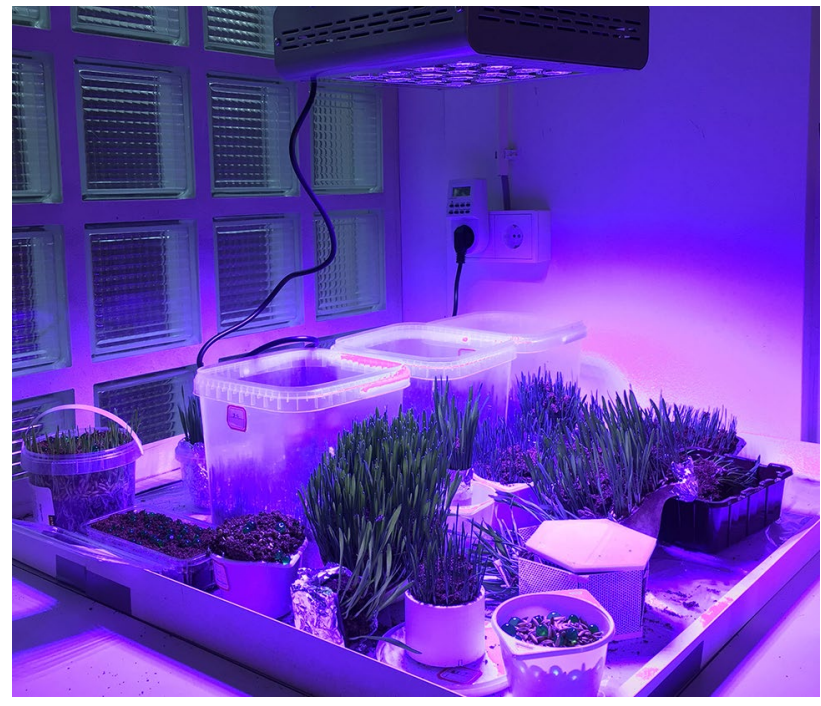

Fig. 4 Additional growing light set-up for growing between April and May

Table 1 Tinkering activity overview

\begin{tabular}{lll}
\hline Growing media & Gravitropism & Barrier \\
\hline Coco coir & Gravity & Form \\
Deep water & Centrifugal force & Direction \\
Shallow water & & Openness \\
Agar gel & & \\
\hline
\end{tabular}

Growing media, gravitropism and barrier are potentially the most influential parameters for our design purpose in consultancy with Scherer

\section{The first set of tinkering activities}

We started tinkering with the roots to get insights on what they can afford and how they behave. We used oat seeds, because of their growing speed and compliance to the predefined grow patterns. Our taxonomy served as a compass to navigate through the most influential material design parameters. We selected, potentially, the most influential parameters for our design purpose in consultancy with Scherer, who had experimented with different roots and growing media. Table 1 provides an overview of the tinkering activities.

The first experiment was conducted to investigate the influence of growing media on roots, which has proved to have a major effect on the optimal strength of the material. Coco coir, water and agar gel are common growing media and have been used by Scherers in her practice.

The next experiment explored root behaviours and morphologies when roots meet different barriers (see "Background" section). In earlier experiments, Scherer had observed that roots grow into fabrics that were incorporated into the template. The ability of roots to grow through a barrier (in this case a piece of fabric) triggered us to explore this potential of roots further, for instance, in building a 3D structure. We documented the results of our observations through mapping the root-barrier interactions, according to the form and direction of the barrier.

In the last experiment, we explored root behaviour when subject to centrifugal force. Scherer had previously tried to grow roots on curved surfaces by growing two halves of a vase separately. But she found that the roots gathered at the bottom of each half, leaving the side parts less colonized. We tested whether centrifugal force would help roots grow towards the side of the vase, creating a 3D seamless form, rather than two separate pieces to be connected afterwards.

\section{Experimental set-up}

The experiments were conducted between April and August 2019 at the Material Experience Lab. Between April and May, due to insufficient natural light, additional
Fig. 5 Centrifugal force experiment set-up (left); 3D-printed moulds for making growing vessels for barrier experiments (right)
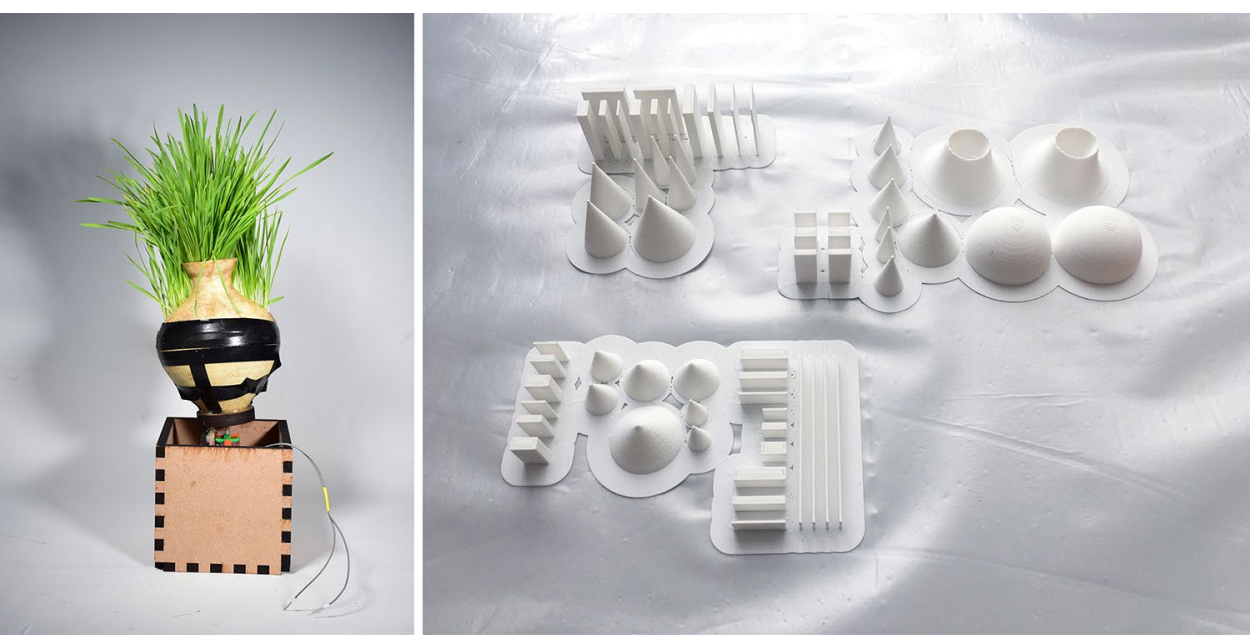
growing light (Fig. 4) (Mars Hydro Pro II Epistar 80, 165 Watt) was used between 6 am and 8 pm to speed up growing; between June and August, and the natural lighting condition and the temperature $\left(20-30{ }^{\circ} \mathrm{C}\right)$ required no additional lighting or air conditioning. The basic set-up included a template, a container where the growing media was placed, and a proper amount of oat seeds (per square millimeter) were sown in the growing container. Whenever agar gel or water was used as the growing media, the root part of the container was covered in darkness [61]. After 7 days, the roots can be harnessed.

In order to explore the impact of barriers on root behaviour, we designed different growing containers and fabricated combining 3D printing and vacuum forming (Fig. 5, right). We also used some existing objects like beads, to place in the container as barriers for quick prototyping. For experimenting with centrifugal force, we created a centrifugal force set-up (Fig. 5, left). We used the vase made by Scherer as two halves with patterns in their inner surface. In our set-up, the two-half of the vase is connected to one container which is fixed on a turning wheel with a spinning motor (spinning speed $=180 \mathrm{rpm}$ ). Oat seeds are planted in the spinning container with inner patterns filled with agar gel and harvested after 7 days (valid spinning time around 3 days).

We screened the processes by observations, taking notes on a material diary, photographs, videos of the process and results. We mapped out the root behaviours and
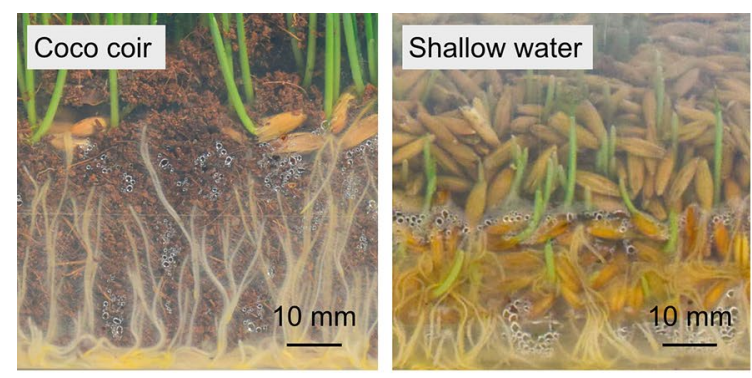

(a)
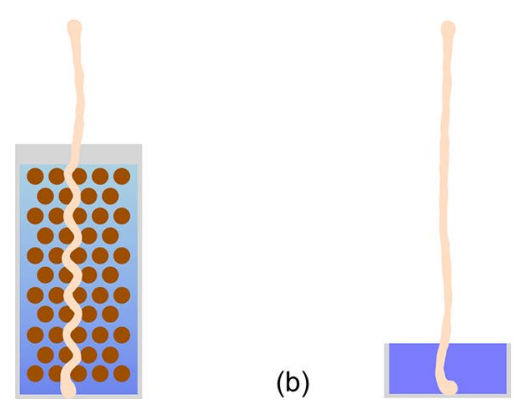

Fig. 6 Root behaviour in different growing media. a Roots grow through coco coir fibres and particles, winding down, elongating fast. b Roots grow to the bottom of the container, elongating more slowly, compared to growing in coco coir. $\mathbf{c}$ Roots stay at a certain length, morphologies and teased out the most promising directions for 3D-form creation and structural stability.

\section{Results}

\section{Mapping the root behaviour}

To show root behaviours as the results from our tinkering activities, for each experiment we present a photograph taken in the tinkering process and a schematic representation depicting the local behaviour, and a description of the root behaviour (Figs. 6, 7, 8).

\section{Root potential for 3D forms}

These first activities showed that roots do behave very sensitively and adapt to the growing environment. Learning that roots can be directed through the means of placing barriers in the growing direction, we have unveiled the root potentials for building 3D forms through binding different elements on their way.

The potentials are twofold. Roots not only are able to not only make a composite with the dried agar membranes, when grown in agar medium (Fig. 9, left), but also can bind the 3D porous barriers in their growing direction (Fig. 9, right). The "glue-ability" affordances of roots in relation to porous structures lead to binding them into $3 \mathrm{D}$ massive form with high compressive strength.
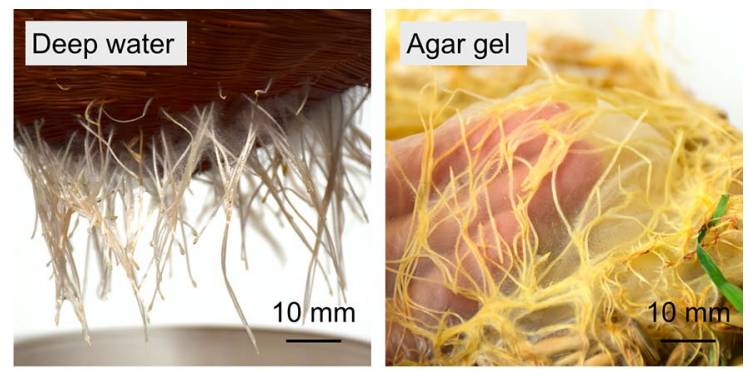

(c)

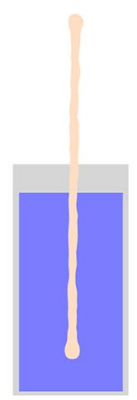

(d)

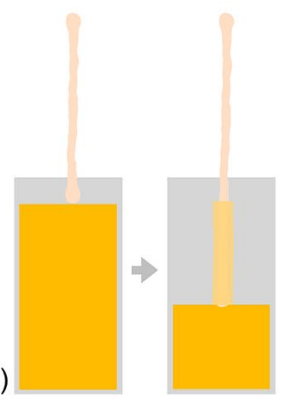

elongating more slowly, compared to growing in coco coir. d Roots consume water in agar gel and leave a dry membrane to embrace roots 
(a) Open barrier

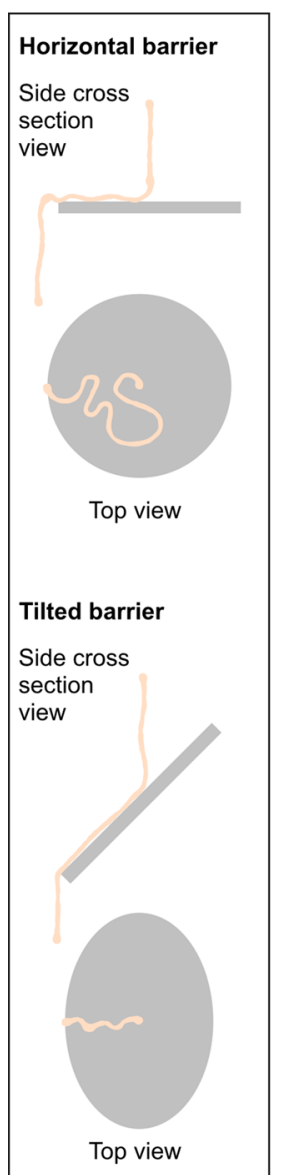

(b) Closed barrier with flat bottom
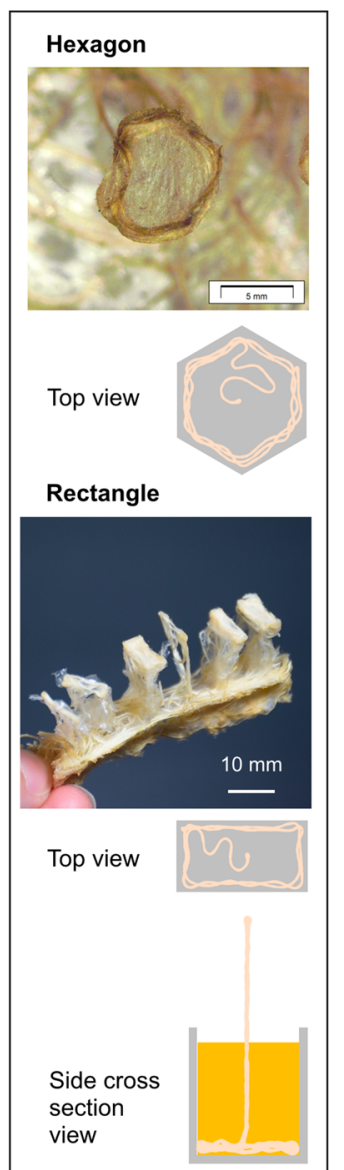

(c) Closed barrier with round bottom
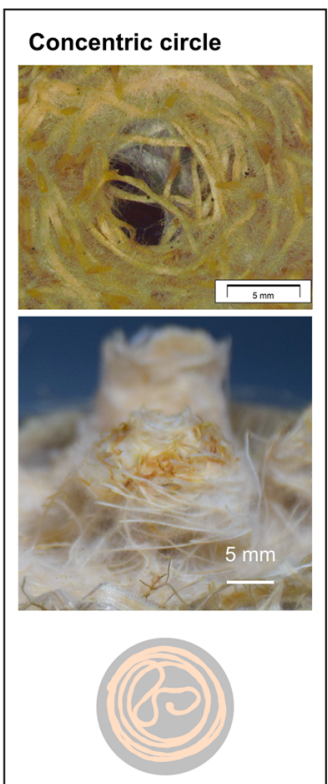

Top view

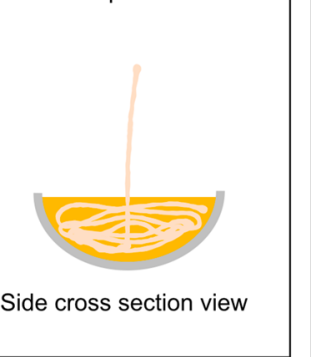

(d) Closed barrier with sharp bottom

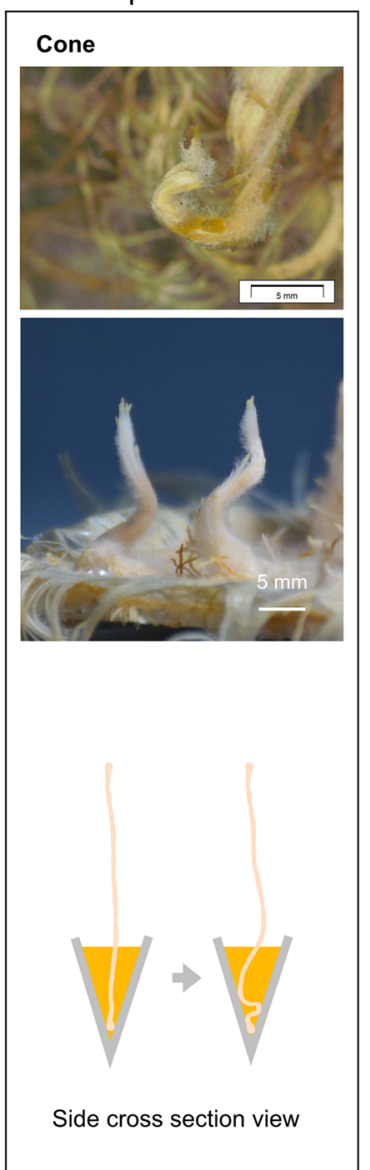

(e) 3D barrier

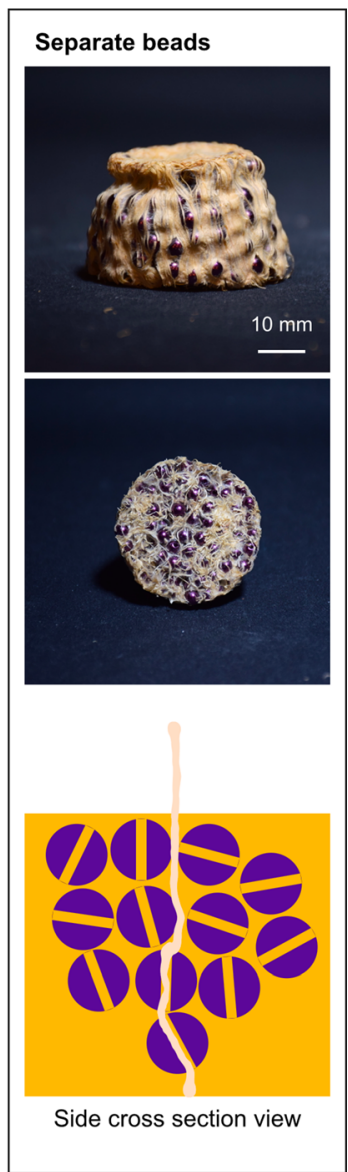

Fig. 7 Root behaviour when they meet barriers, of different shapes, angles and dimensions (3D beads). a When roots grow and meet horizontal barrier, they grow in all directions until they reach the edge and find the way down again; when they meeting tilted barrier, they meander downwards until finding the edge. b When roots meet closed barrier with flat bottom, they go straight to the surface of the barrier and then go from the touching point to the edge, forming the perim- eter of the barrier shape. $\mathbf{c}$ When roots meet closed barrier with round bottom, they form concentric circles and 3D form. $\mathbf{d}$ When roots meet closed barrier of sharp bottom, they get stuck at the tip of the barrier and then pushed back, becoming a curved slim form. e When roots meet 3D barrier, they grow in between the 3D beads or through the holes in the axis of the beads; in the end, roots connect all the beads together
Herein, we question: how do we design a porous structure to elevate the roots' glue-ability potential to create a product? In the next section, we will present the digitally designed and engineered beads to further explore roots' affordances and take a closer look at the micro-interactions between roots and the porous structure.

\section{Designing the bead: digital biofabrication with plant roots}

According to the presented root behaviour, if we could design proper barriers for roots to grow through, roots can build a composite 3D form together with agar gel and the porous barriers. In order to design and optimize the barrier, we picked a possible design direction for the ultimate product: a low stool, which provided us with a specific material performance we expect from the grown composite structure to fulfil. In the related work, we have seen designers 3D print scaffolds and boundaries to enhance the strength of mycelium chair. Here, we explored 3D printing and parametric design of the single unit that roots are going to connect. In the end, a low sitting stool has been collectively made from the growing of roots and 600 porous beads. The stool is fully functional with load bearing, proving a fabrication technique with roots for creating 3D massive products.

The design requirements for the beads included: (1) to be light enough for the roots to hold together; (2) to be porous; and (3) to be bio-based to be compliant with "root" material, leading to the final stool that withstands the weight of an average person while providing basic comfort for sitting on. 
Fig. 8 Root behaviour when they are influenced by centrifugal force. In the presence of centrifugal force, roots grow towards the sides of the vase. Without centrifugal force, roots grow only towards gravity
Fig. 9 3D forms grown by roots (left); 3D from co-created by roots and 3D barriers (right)
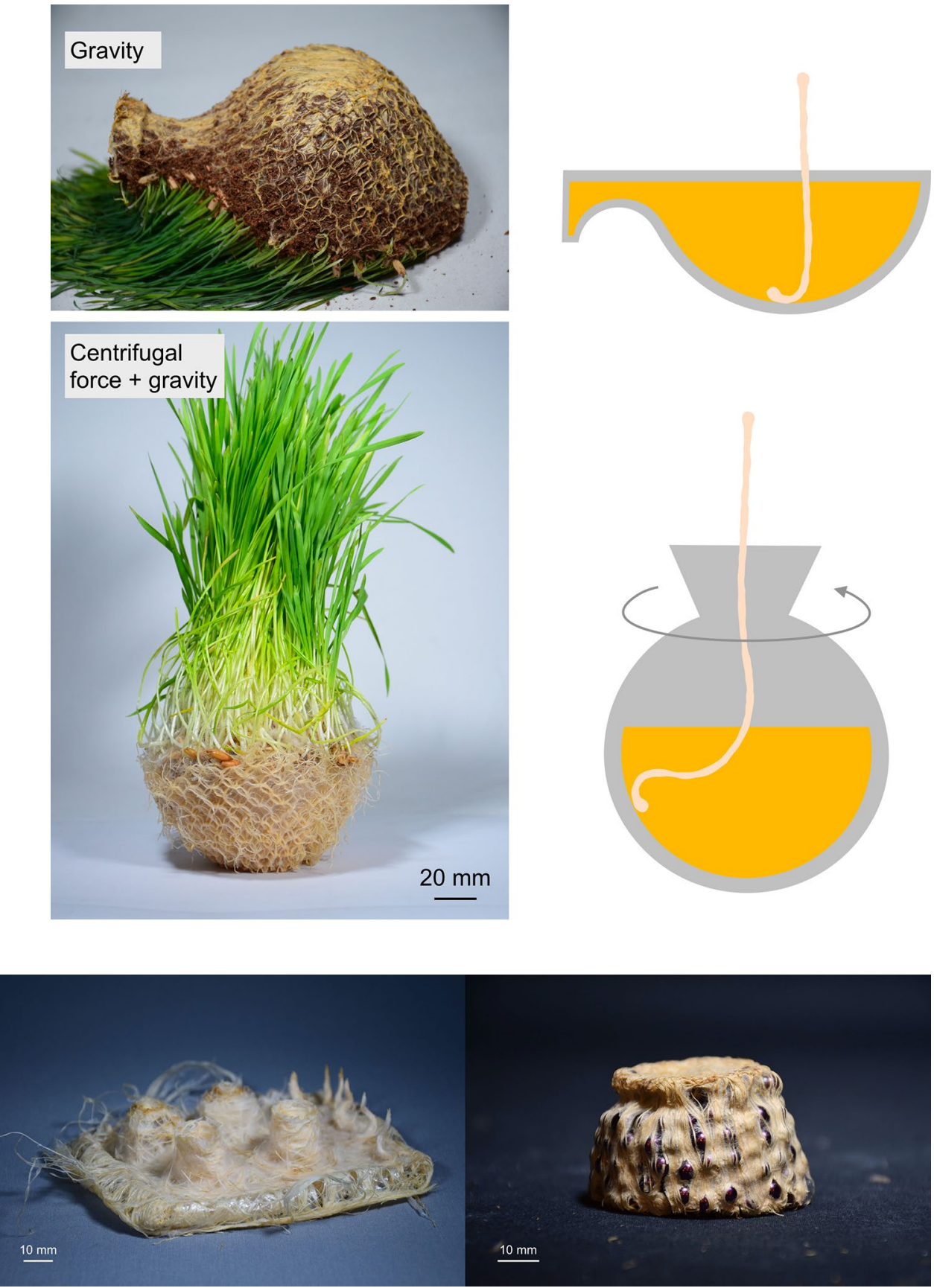

To make the bead lightweight and robust, we have focused on porous structures that are commonly seen in nature, such as trabecular bone. Bone-like structure has been an appealing option as infill for additive manufacturing [45]. The spongy trabecular bone adapts itself to the mechanical load [40] from a natural optimization process. The resulting composition is lightweight, resistant, robust with respect to force variations and damage-tolerant [45].

An Ultimaker 3D printer with polylactic acid (PLA-a type of bioplastic derived from renewable resources) filament was used to fabricate the beads. The beads were then prepared in the agar gel where roots grew. The 3D-printed PLA structure was generated by a parametric modelling algorithm in Grasshopper (Fig. 11), the parametric design plugin of Rhinoceros (3D modelling software). We chose two types of porous structure: radiative (Fig. 10B(a)) and hollow (Fig. 10B(c)). The radiative porous structure was found to be too stiff, while the hollow structure was lighter and more flexible. In Fig. 12, the growing/binding process is shown through a photograph taken on the fourth day of growth. The agar gel was consumed where roots prevailed and the porous PLA beads were occupied and sewn 
Fig. 10 A Overview of the bead design and optimization process. B $(a)$ the radiative boneinspired porous structure; $(b)$ roots grown into the radiative bone-inspired porous structure; (c) the hollow bone-inspired porous structure and $(d)$ roots grown into the hollow boneinspired porous structure
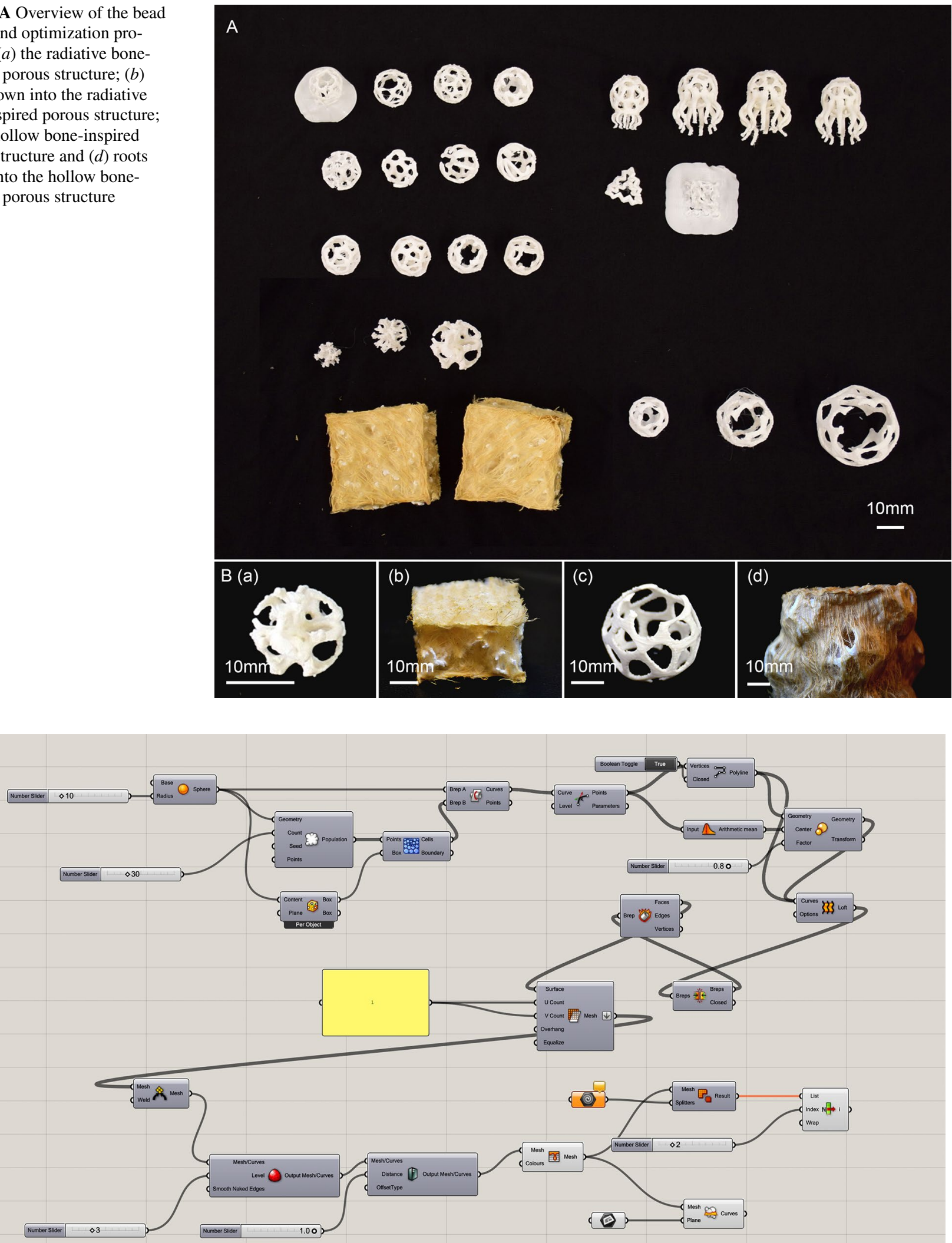

Fig. 11 Screenshot of a parametric design process: to create the hollow porous structure 


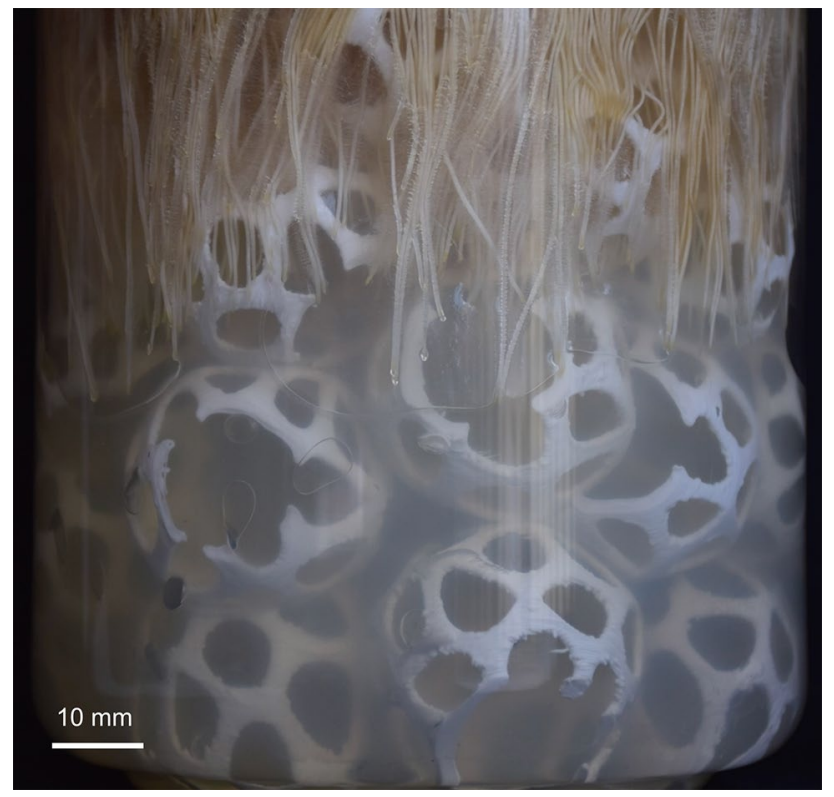

Fig. 12 Glimpse into the growing process and glue-ability of roots

together. Figure 13 shows the final low stool fabricated by roots.

\section{Discussion}

This research provides a systematic approach to understand plant root behaviour in designing and fabricating 3D forms in product design. The material-driven design process unveiled new affordances of roots in binding 3D-printed porous structure, supported by research on roots' responsive behaviour and digital biofabrication. We showed that plant roots are able to bind together the growing media and the digital fabricated barriers in the growing process to build a predefined 3D form. This "glue-ability" affordance of roots, which has also been brought forth in designing with other living organisms such as mycelium [see, for example, MycoTEX, Aniela Hoitink, NEFFA; Chair made with Mycelium by Officina corpuscoli], can be further explored in future studies to tailor material properties in a more controlled manner through the designing and optimization of porous structures. For example, the mechanism of roots binding porous structures can be more accurately described with a mathematical model that may be useful for predicting root path and the link in local microstructures created by roots and 3D-printed structures, to achieve well-defined, local material properties [48].

Looking at root behaviours and materials potential found in this research, we could further show the roots dynamic interactions and intelligent adaptation to the growing space, which will bring new challenges to design, for example, to think creatively for constructing a most optimum habitat for the living [48]. Further implications for design and human-computer interaction research could expand on the affordances of root as an intelligent agent-e.g. by carrying information/signal according to environment changes, or acting as "information" carriers according to their locomotion.

We hope that our work inspires and informs biodesigners towards pushing the boundaries between the living and nonliving, nature and manmade and computational and biological intelligence.

\section{Conclusion}

This research systematically explores plant roots as productive and intelligent agents for fabricating $3 \mathrm{D}$ objects by creating hybrid materials of the living and non-living through digital biofabrication. Taking a material-driven design approach, the paper presents our tinkering activities with roots and points out the glue-ability potential of roots for building 3D structures through binding different elements
Fig. 13 Low stool grown by roots, connecting 600 hollow, parametrically optimized porous beads
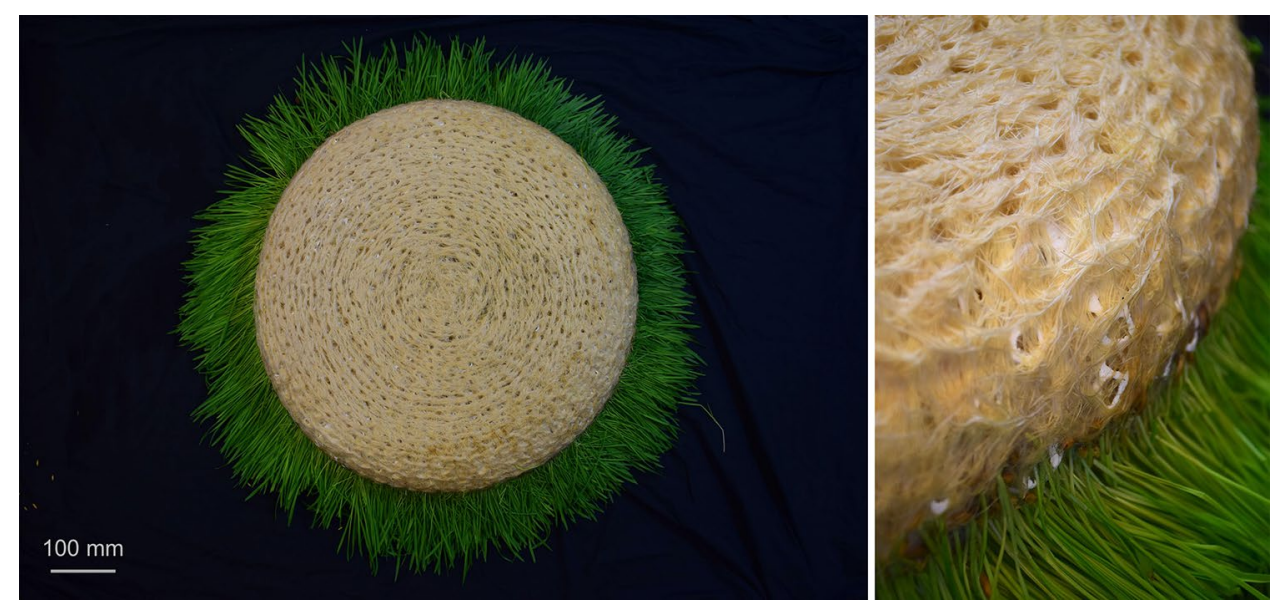
on their way. The presented mapping of the root behaviour and our systematic approach can serve as an initial guideline for biodesign communities interested in further explorations not only with plant roots but other living organisms. Future research is suggested on plant root behaviour to leverage on the uncovered affordances in creating an expanded range of material properties such as elasticity and designing interactive systems, when they are kept alive.

Author contributions Jiwei Zhou participated in conceptualization, investigation, writing of the original draft and visualization. Bahareh Barati contributed in conceptualization, methodology, writing, review and editing. Jun $\mathrm{Wu}$ participated in methodology, writing, review and editing, and supervision. Diana Scherer provided the original technique of experiments, reviewing. Elvin Karana participated in conceptualization, methodology, writing, review and editing and supervision. All authors have read and approved the final manuscript and, therefore, have full access to all the data in the study and take responsibility for the integrity and security of the data.

\section{Compliance with ethical standards}

Conflicts of interest All authors declare that they have no conflict of interest.

Human and animal right statement This article does not contain any studies with human or animal subjects performed by any of the authors.

Open Access This article is licensed under a Creative Commons Attribution 4.0 International License, which permits use, sharing, adaptation, distribution and reproduction in any medium or format, as long as you give appropriate credit to the original author(s) and the source, provide a link to the Creative Commons licence, and indicate if changes were made. The images or other third party material in this article are included in the article's Creative Commons licence, unless indicated otherwise in a credit line to the material. If material is not included in the article's Creative Commons licence and your intended use is not permitted by statutory regulation or exceeds the permitted use, you will need to obtain permission directly from the copyright holder. To view a copy of this licence, visit http://creativecommons.org/licenses/by/4.0/.

\section{References}

1. Myers W (2012) Biodesign. Nature, science, creativity. Thames \& Hudson, High Holborn, UK, p 288

2. Ginsberg AD, Calvert J, Schyfter P, Elfick A, Endy D (2014) Synthetic aesthetics: investigating synthetic biology's designs on nature. MIT Press, Cambridge

3. Collet C (2017) Grow-made textiles. In: Karana E, Giaccardi E, Nimkulrat N, Niedderer K, Camere S (eds) Alive active adaptive: proceedings of EKSIG2017 (pp. 24-37). International conference on experiential knowledge and emerging materials, June 19-20, Delft, The Netherlands

4. Camere S, Karana E (2018) Fabricating materials from living organisms: an emerging design practice. J Clean Prod 186:570584. https://doi.org/10.1016/j.jclepro.2018.03.081

5. Mironov V, Trusk T, Kasyanov V, Little S, Swaja R, Markwald R (2009) Bio-fabrication: a 21st century manufacturing paradigm. Biofabrication 1(2):022001
6. Holt GA, McIntyre G, Flagg D, Bayer E, Wanjura JD, Pelletier MG (2012) Fungal mycelium and cotton plant materials in the manufacture of biodegradable molded packaging material: evaluation study of select blends of cotton byproducts. J Biobased Mater Bioenergy 6(4):431-439. https://doi.org/10.1166/jbmb.2012.1241

7. Ginsberg AD, Chieza N (2018) Editorial: Other biological futures. J Des Sci. https://doi.org/10.21428/566868b5

8. Lelivelt RJJ, Lindner G, Teuffel P, Lamers H (2015) The production process and compressive strength of Mycelium-based materials. In: First international conference on bio-based building materials

9. Pavlovich MJ, Hunsberger J, Atala A (2016) Biofabrication: a secret weapon to advance manufacturing, economies, and healthcare. Trends Biotechnol 34(9):679-680. https://doi.org/10.1016/j. tibtech.2016.07.002

10. Fujii H, Yoshida K, Sugimura K (2016) Research and development strategy in biological technologies: a patent data analysis of Japanese manufacturing firms. Sustainability 8(4):351

11. Holland I, Logan J, Shi J, McCormick C, Liu D, Shu W (2018) 3D biofabrication for tubular tissue engineering. Bio-Des Manuf 1(2):89-100. https://doi.org/10.1007/s42242-018-0013-2

12. Elbaz A, He Z, Gao B et al (2018) Recent biomedical applications of bio-sourced materials. Bio-Des Manuf 1:26-44

13. Lode A et al (2015) Green bioprinting: fabrication of photosynthetic algae-laden hydrogel scaffolds for biotechnological and medical applications. Eng Life Sci 15(2):177-183. https://doi. org/10.1002/elsc.201400205

14. Liu $X$ et al (2017) 3D printing of living responsive materials and devices. Adv Mater. https://doi.org/10.1002/adma.201704821

15. Smith RSH et al (2020) Hybrid living materials: digital design and fabrication of 3D multimaterial structures with programmable biohybrid surfaces. Adv Funct Mater 30(7):1-14. https ://doi.org/10.1002/adfm.201907401

16. Jian H, Wang M, Wang S, Wang A, Bai S (2018) 3D bioprinting for cell culture and tissue fabrication. Bio-Des Manuf 1(1):4561. https://doi.org/10.1007/s42242-018-0006-1

17. Ozbolat IT, Yu Y (2013) Bioprinting toward organ fabrication: challenges and future trends. IEEE Trans Biomed Eng 60(3):691-699. https://doi.org/10.1109/TBME.2013.2243912

18. Ye J, Chu T, Chu J, Gao B, He B (2019) A versatile approach for enzyme immobilization using chemically modified 3D-printed scaffolds. ACS Sustain Chem Eng 7(21):18048-18054. https:// doi.org/10.1021/acssuschemeng.9b04980

19. Aspling F, Wang J, Juhlin O (2016) Plant-computer interaction, beauty and dissemination. In: ACI '16: Proceedings of the third international conference on animal-computer interaction. https ://doi.org/10.1145/2995257.2995393

20. Weil F (2014) Human-plant interface. http://archive.derhess.de/ blog.derhess.de/human-plant-interfaces/index.html. Accessed 09 June 2020

21. O'Nascimento R (2010) Plants parlantes. http://www.onasc imento.com/plantas-parlantes. Accessed 09 June 2020

22. MSHR (2011) Baumarktmusik. http://www.mshr.info/MSHRq uioche.html. Accessed 09 June 2020

23. Wuschitz S (2012) Mosszillator. http://grenzartikel.com/proje $\mathrm{cts} / \mathrm{p}=841$. Accessed 09 June 2020

24. Martinez C (2013) Frishasin pijamas. https://vimeo.com/66130 373. Accessed 09 June 2020

25. Dogane Y (2011) Plantron. http://www.spoon-tamag o.com/2012/03/29/yuji-dogane-radio-active-plantron/. Accessed 09 June 2020

26. Henriques I (2011) Jurema action plant. https://ivanhenriques. com/works/jurema-action-plant/. Accessed 09 June 2020

27. Masaoka M (2020) Pieces for plants. http://miyamasaoka.com/ work/2006/pieces-for-plants-gallery-installation/. Accessed 09 June 2020 
28. Poupyrev I, Schoessler P, Loh J, Sato M (2012) Botanicus interacticus: interactive plants technology. In: ACM SIGGRAPH 2012 emerging technologies, SIGGRAPH'12. https://doi. org $/ 10.1145 / 2343456.2343460$

29. Seo JH, Sungkajun A, Suh J (2015) Touchology: towards interactive plant design for children with autism and older adults in senior housing. https://doi.org/10.1145/2702613.2732883

30. Manzella V et al (2013) Plants as sensing devices. https://doi. org/10.1145/2517351.2517403

31. Cheok AD, Kok RT, Tan C, Fernando ONN, Merritt T, Sen JYP (2008) Empathetic living media. https://doi.org/10.1145/13944 45.1394495

32. Hamidi F, Baljko M (2014) Rafigh: a living media interface for speech intervention. https://doi.org/10.1145/2556288.2557402

33. Chien JT, van Guimbretière F, Rahman T, Gay G, Matthews M (2015) Biogotchi! An exploration of plant-based information displays. https://doi.org/10.1145/2702613.2732770

34. Holstius D, Kembel J, Hurst A, Wan PH, Forlizzi J (2004) Infotropism: living and robotic plants as interactive displays

35. Portocarrero E, Dublon G, Paradiso J, van Bove M (2015) ListenTree: audio-haptic display in the natural environment. https://doi. org/10.1145/2702613.2725437

36. Kuribayashi S, Sakamoto Y, Tanaka H (2007) I/O plant: a tool kit for designing augmented human-plant interactions. In: Conference on human factors in computing systems-proceedings, pp 2537-2542. https://doi.org/10.1145/1240866.1241037

37. Kawakami A, Tsukada K, Kambara K, Siio I (2011) PotPet: petlike flowerpot robot. https://doi.org/10.1145/1935701.1935755

38. Park S, Oh S, Hahn M (2008) Emoti-pot: the interaction design of an affective flowerpot. In: Proceedings of advances in computer entertainment technology

39. Hwang S, Lee K, Yeo W (2010) My green pet. https://doi. org/10.1145/1810543.1810573

40. Wolff J (1893) Das gesetz der transformation der knochen. DMWDeuts Med Wochenschr 19(47):1222-1224

41. Schury F, Stingl M, Wein F (2012) Efficient two-scale optimization of manufacturable graded structures. SIAM J Sci Comput 34(6):B711-B733

42. Wang $X$ et al (2016) Topological design and additive manufacturing of porous metals for bone scaffolds and orthopaedic implants: a review. Biomaterials 83:127-141

43. Coelho PG, Fernandes PR, Rodrigues HC, Cardoso JB, Guedes JM (2009) Numerical modeling of bone tissue adaptation-a hierarchical approach for bone apparent density and trabecular structure. J Biomech 42(7):830-837

44. Huiskes R, Ruimerman R, van Lenthe GH, Janssen JD (2000) Effects of mechanical forces on maintenance and adaptation of form in trabecular bone. Nature 405(6787):704-706

45. Wu J, Aage N, Westermann R, Sigmund O (2017) Infill optimization for additive manufacturing-approaching bone-like porous structures. IEEE Trans Vis Comput Graph 24(2):1127-1140
46. Heppenstall AJJ, Crooks AT, See LM, Batty M (2012) Agent-based models of geographical systems. AgentBased Models Geogr Syst 164(2011):1-759. https://doi. org/10.1007/978-90-481-8927-4

47. Neri Oxman CG, Laucks J, Kayser M (2017) Silk Pavilion : a case study in fibre-based digital fabrication. In: Uribe CCGN (ed) Fabricate: negotiating and making. GTA Verlag, Zurich

48. Oxman N, Laucks J, Kayser M, Uribe CCDG, Duro-Royo J (2013) Biological computation for digital design and fabrication: a biologically-informed finite element approach to structural performance and material optimization of robotically deposited fibre structures. In: eCAADe 2013: computation and performanceproceedings of the 31 st international conference on education and research in computer aided architectural design in Europe, Delft, The Netherlands, September 18-20, vol 1, pp 585-594. http:// repository.tudelft.nl/view/conferencepapers/uuid:241873a0-ad1443f8-a135-e2c133622c2f/

49. Yao L et al (2015) Biologic: Natto cells as nanoactuators for shape changing interfaces. In: Conference on human factors in computing systems-proceedings, vol 2015, pp 1-10. https://doi. org/10.1145/2702123.2702611

50. Edelmann HG (2018) Graviperception in maize plants: is amyloplast sedimentation a red herring? Protoplasma 255(6):1877-1881

51. Darwin C, Darwin F (1881) The power of movement in plants. D. Appleton and Co., New York

52. Trewavas A (2002) Plant intelligence: mindless mastery. Nature 415(6874):841

53. Sievers A, Braun M, Monshausen GB (2002) The root cap: structure and function. In: Plant roots. CRC Press, London, pp 79-103

54. Beams HW, King RL (1939) The effect of centrifugation on plant cells. Bot Rev 5(2):132

55. Tan TH, Silverberg JL, Floss DS, Harrison MJ, Henley CL, Cohen I (2015) How grow-and-switch gravitropism generates root coiling and root waving growth responses in Medicago truncatula. Proc Natl Acad Sci 112(42):12938-12943

56. Smith S, de Smet I (2012) Root system architecture: insights from Arabidopsis and cereal crops. The Royal Society, London

57. Brunner I, Herzog C, Dawes MA, Arend M, Sperisen C (2015) How tree roots respond to drought. Front Plant Sci 6:547

58. Karana E, Barati B, Rognoli V, Zeeuw van der Laan A (2015) Material driven design (MDD): a method to design for material experiences. Int J Des 9(2):35-54

59. Barati B, Karana E (2019) Affordances as materials potential: what design can do for materials development. Int J Des 13(3): 105-123

60. Karana E, Blauwhoff D, Hultink EJ, Camere S (2018) When the material grows: a case study on designing (with) mycelium-based materials. Int J Des 12(2):119-136

61. Xu W et al (2013) An improved agar-plate method for studying root growth and response of Arabidopsis thaliana. Sci Rep 3:1273 